\title{
A Probabilistic Approach for Prognosis of Battery Pack Aging
}

\author{
Chin Yao Chang ${ }^{\mathrm{a}}$, Punit Tulpule ${ }^{\mathrm{b}, *}$, Giorgio Rizzoni ${ }^{\mathrm{b}}$, Wei Zhang ${ }^{\mathrm{a}}$, Xinyu Du ${ }^{\mathrm{c}}$ \\ ${ }^{a}$ Dept. of Electrical Engineering, The Ohio State University, Columbus, $\mathrm{OH}$ \\ ${ }^{b}$ Center for Automotive Research, The Ohio State University, Columbis, OH \\ ${ }^{c}$ General Motors Research and Development, Warren MI
}

\begin{abstract}
A probabilistic framework is developed for the prognosis of battery packs. It is demonstrated using aging campaign data, that aging models alone may not be sufficient for aging prognosis, and aging model parameter estimation may further improve the accuracy of prognosis. A systematic framework that extends the aging models to battery pack aging and prognosis still remains challenging. We propose a framework that bridges the gap in cell and pack aging prognosis in a probabilistic sense, and further improves the prognosis by estimating the aging model parameters for the pack. The framework is versatile for various applications because it is not restricted to a specific cell chemistry, or a type of aging model. In addition, the proposed framework could distinguish more aged cells as compared to other cells in the pack. Numerical examples are provided to demonstrate the effectiveness of the proposed framework.
\end{abstract}

Keywords: Li-Ion Battery packs, prognosis, estimation, PHEV

\section{Introduction}

Aging of dynamical systems is defined as the loss of functionality over time. Understanding of aging phenomena is critical for predicting the Remaining Use-

\footnotetext{
* Corresponding author

Email addresses: chang.981@osu.edu (Chin Yao Chang), tulpule.3@osu.edu (Punit Tulpule ), rizzoni.1@osu.edu (Giorgio Rizzoni), zhang.491@osu.edu (Wei Zhang), xinyu.du@gm.com (Xinyu Du)

Preprint submitted to Journal of Power Sources

December 25, 2016
}

(C) 2017. This manuscript version is made available under the Elsevier user license http://www.elsevier.com/open-access/userlicense/1.0/ 


\begin{tabular}{|c|c|c|c|}
\hline \multicolumn{4}{|c|}{ Nomenclature } \\
\hline$[n]$ & a sequence $1,2, \ldots, n$ & $R$ & ideal gas constant \\
\hline$\alpha$ & $\begin{array}{l}\text { Collection of aging model pa- } \\
\text { rameters }\end{array}$ & Ratio & $\begin{array}{l}\text { Time fraction of charge de- } \\
\text { pleting }\end{array}$ \\
\hline $\bar{X}$ & $\begin{array}{l}\% \text { capacity loss for a battery } \\
\text { to reach the end of life }\end{array}$ & $S$ & Capacity of a battery cell \\
\hline$\hat{X}$ & Estimated \% capacity loss & $S o C$ & State of charge \\
\hline $\mathbb{1}_{\mathcal{A}}$ & Indicator function for set $\mathcal{A}$ & $T$ & Temperature \\
\hline$\Omega$ & Probability distribution & $V$ & Terminal voltage \\
\hline & function of $w$ & $V_{o c}$ & Open circuit voltage \\
\hline$A h$ & $\begin{array}{l}\text { Accumulated Ampere-hour } \\
\text { current throughput }\end{array}$ & $w$ & $\begin{array}{l}\text { Estimation error of pack ca- } \\
\text { pacity }\end{array}$ \\
\hline $\begin{array}{l}D^{0} \\
E_{a c}\end{array}$ & $\begin{array}{l}\text { Initial distribution of } \alpha \\
\text { Activation energy }\end{array}$ & $X_{c}$ & $\%$ capacity loss of a pack \\
\hline$I$ & Current & $x_{c}$ & $\%$ capacity loss of a cell \\
\hline$p_{i}$ & $\begin{array}{l}\text { Probability of cell } i \text { being } \\
\text { most aged }\end{array}$ & $\begin{array}{l}X_{r} \\
x_{r}\end{array}$ & $\begin{array}{l}\% \text { resistance loss of a pack } \\
\% \text { resistance loss of a cell }\end{array}$ \\
\hline
\end{tabular}

ful Life (RUL) and for system design. Prognosis allows prediction of RUL using 5 current state of the system, aging dynamics and future operating conditions. Prognosis for interconnected systems poses challenges due to the aging propagation among the components and other system interactions. Aging of one element may influence aging in the other elements, leading to faster aging of the overall system. A battery pack consists of battery cells, cooling system, and 10 Battery Management System (BMS). Life of the battery pack affects the lifetime cost of the vehicle, which includes servicing, maintenance and equipment replacement costs. Understanding the aging phenomena of the battery pack has 
been a research topic in the automotive industry for a couple of decades $[1,2,3]$. Prognosis methods in general are not new to the automotive industry. For example, life of engine oil is dynamically computed using actual in-use vehicle conditions and that information is used to predict the time until the next oil change. Similarly, prognosis of battery packs in electrified vehicles is important to avoid sudden power loss. Furthermore, prognostic capabilities may enable life extending functions in the vehicle control systems.

Various types of aging models have been developed and played a pivotal role in prognosis $[4,5,6,7]$. However, these high fidelity aging model are nonlinear and involve many model parameters required to be identified. The aging phenomena is attributed to a complicated electrochemical reaction mechanism. In order to develop an aging model for a pack for on-board prognosis, a control oriented model is necessary $[8,9,10,11]$.

Past research at the Center for Automotive Research (CAR), at the Ohio State University (OSU) has established an aging model for lithium-ion pouch cells containing blended spinel and layered-oxide positive electrodes. These aging models were then extended for packs by appropriate consideration of electrical and thermal balancing as well as the differences among individual cells [12]. A semi-empirical aging model is adopted in the work to reach a balance between model complexity, computational efficiency and physical understanding of the aging mechanism for a cell. Such a model may be implemented for pack-level prognosis when the aging model of every cell in the pack is known. However, the manufacturing variability may cause heterogeneity between the interconnected cells, making identification of the aging model for all the cells challenging. One way of addressing this issue is to consider aging model parameters as the state variables and implement dynamic estimation methods such as Extended Kalman Filter (EKF) or Nonlinear Predictive Filter (NPF) to estimate both State Of 40 Health $(\mathrm{SOH})$ and the aging model. However, in most experimentally validated aging models $[13,14]$, the $\mathrm{SOH}$ appears as a nonlinear static function of measured or estimated variables, such as State Of Charge (SOC) or temperature. Those filtering techniques may not be directly applicable for on-board prognosis 
due to computational complexity. These approaches require a separate filter for each cell or module in series. Another approach is to identify the aging model on line using machine learning and data driven algorithms, for example, Artificial Neural Networks (ANNs) $[15,16]$.

In this paper, we propose a model based estimation framework that is specifically developed for the algebraic aging models in order to improve both accuracy and computational complexity. The proposed framework updates the aging model of the cells with only the knowledge of SOH and SOC estimation of the entire pack. Cell-to-cell heterogeneity due to manufacturing variability and exposure to different temperatures in a pack can be probabilistically quantified using measurements or estimations of physical parameters of cells. The aging model of the pack can then be derived by combining cell aging models in probabilistic sense. The proposed framework is suitable for on-board prognosis of a Plug-in Hybrid Electric Vehicle (PHEV) battery pack. It is not restricted to any specific cell chemistry or a type of aging model. In addition, the proposed framework updates the parameters of the aging model of the pack if physical parameter measurements and estimations of SOC and $\mathrm{SOH}$ are available, which is generally true in practical usage of PHEV. It is worth mentioning that the probability of one cell being most aged (or the probability of being the "weakest" cell) is naturally included in the framework. This information is valuable to improve the BMS control scheme, rebuilding the battery pack, and replacing aged modules to prolong the life time of the pack. Hence, we believe the paper makes important contributions that are not available in the literature:

1. extension of the cell-level aging model to pack aging model in a probabilistic sense

2. identification of a the semi-empirical aging model that can be used with measurements available in a typical production PHEV

3. identification of the most aged cell in a battery pack

The rest of the paper is organized as follows. In section 2, we review the aging model of a cell. A motivating example based on experiment data is also pro- 
vided to illustrate the necessity of aging model parameter estimation. Section 3 establishes the probabilistic framework to aging characteristics of the battery packs. Simulation studies and experiments are demonstrated in Section 4 to show the effectiveness of the proposed method.

\section{Aging Model - Background}

In this section, we will first review typical aging models for a battery cell, and how degradation of single cell affects the aging of a pack. A realistic method for identifying the aging model for battery cell is also described. Using single cell aging campaign data, we demonstrate that the aging model parameter estimation improves the predictive capabilities of the aging model. This example provides motivation for pack level aging model parameter estimation to improve prognosis of a pack.

\subsection{Aging Model of Single Cell}

The aging of a battery cell is characterized by the capacity fade and resistance growth. For the aging attributed to resistance growth, we will only focus on the ohmic resistance instead of detailed second order equivalent resistance model $[5,13]$. Let $x_{c}$ and $x_{r}$ be the percentage capacity loss and resistance growth respectively. The aging model of cell $i$ can be written in the following form in general

$$
\left\{\begin{array}{l}
x_{c, i}(t)=f_{c}\left(z_{i}, \alpha_{i}, t\right) \\
x_{r, i}(t)=f_{r}\left(z_{i}, \alpha_{i}, t\right)
\end{array}, \quad z_{i}=\left[S O C_{i}, T_{i}, V_{i}, I_{i}\right]\right.
$$

where $T_{i}$ is the battery internal temperature, $V_{i}$ is the terminal voltage, $I_{i}$ ${ }_{95}$ is the input current, and $\alpha_{i} \in \mathbb{R}^{n}$ is the vector collecting the aging model parameters [17]. The $z_{i}$, also called as stress factors, are time dependent, and aging of the cell depends on the time history of these inputs. However, it has been shown using Palmgren-Miner rule that the aging is cumulative and does 
not depend on the cycling sequence, provided certain conditions are met [18]. Hence, the history of $z_{t}$ is captured in cumulative sense. For example $S O C(t)$ may appear as $\min _{\tau \in[0, t)}(S O C(\tau))$, and current usually appears as cumulative charge throughput.

\subsection{Pack-Cell Aging Relation}

As shown in Fig. 1, a common commercial battery pack in PHEV has the strings of parallel (SP) cells interconnection to provide desired current as well as power. However, cell balancing control is typically not implemented for each cell in the elements of parallel cells, since on-board estimation of current split is not trivial. Hence, the cells connected in parallel can be considered as one element [19] for on-board prognosis application. We can therefore equivalently study a string of cells interconnection shown in Fig. 4 . Let $N$ be the number of elements (cells) in the string. If the cell balancing is passive, then the percentage capacity loss of the entire pack $X_{c}$ is given as [20]

$$
X_{c}=\max _{i \in[N]} x_{c, i},
$$

where the capacity aging of the pack is characterized by the one associated with the most aged (weakest) cell in the string [21]. Similarly, the percentage resistance growth of the pack $X_{r}$ is [20]

$$
X_{r}=\max _{i \in[N]} x_{r, i} .
$$

The resistance of the pack is characterized by the cell with the largest resistance. Due to the direct relation between SOC and capacity, SOC of the pack is defined similarly $[20]$.

$$
\mathrm{SOC}=\min _{i \in[N]} \mathrm{SOC}_{i} .
$$

Readers are referred to [19] for more details about the pack-cell relation in Eq. (2-4). In an ideal case where the aging model of every cell in the pack is well 


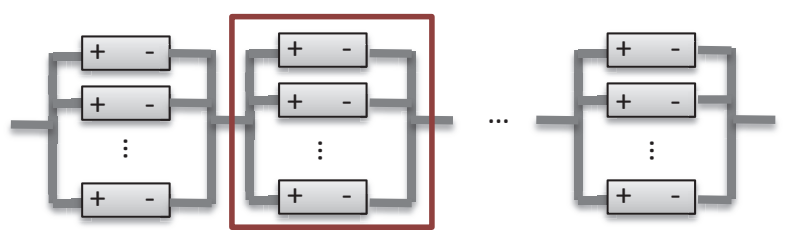

Figure 1: Strings of parallel (SP) cells interconnection. The parallel connected cells can be considered as a single element in analysis due to passive balance control in the pack.

identified, and time history of inputs $z_{i}$ are available with no uncertainty, $X_{c}$ and $X_{r}$ can be directly computed by the pack-cell relations in Eq. (2-4) . However, $\alpha_{i}$ is different between cells due to the manufacture difference. The identification of $\alpha_{i}$ is challenging due to the limited measurements available for cells. For example, the capacity estimation has orders of magnitude larger sampling time as compared to the sampling time step for current, temperature and voltage measurements. Also, in a typical PHEV the capacity measurement/estimation is only available over pack, but not over each single cell. In addition, aging is characterized by nonlinear algebraic equations (1) instead of dynamical models, hence common filtering techniques cannot be directly used. Therefore, we need to develop a parameter estimation algorithm for interconnected systems.

In rest of the paper, we will only focus on the capacity aging because the capacity loss and resistance growth have similar aging models and pack-cell aging relationship. The results for the capacity aging model can be directly applied to the resistance aging.

\subsection{Motivating Example for Parameter Estimation}

In this subsection, we will use experimental data to demonstrate the necessity of parameter estimation. The motivating example suggests that even for the single cell case, a small error in the aging model parameter calibration can lead to a unsatisfactory prognosis [22]. The aging model of a single cell is typically calibrated using laboratory test beds under controlled environmental 
conditions. The calibrated model obtained from the aging campaigns is then used for SOH estimation and PHM. Due to cell-to-cell variations and modeling approximations, the aging model parameters collected in $\alpha_{i}$ are uncertain. A small error of the parameters may lead to a large error in the prognosis. In this part of the research, we have performed sensitivity analysis on the single cell model in order to demonstrate that even for single cell, the aging model alone is not sufficient for long range prognosis. We then use some standard parameter and state estimation algorithms to show that the parameter estimation improves prognosis. Development and calibration of single cell model is done using aging campaigns performed at Center for Automotive Research (CAR), at the Ohio State University (OSU).

\subsubsection{Single Cell Capacity Fade Model}

The capacity fade due to charge-discharge cycling of Li-Ion cells is enhanced by cell skin temperature, cumulative charge throughput, minimum SOC, charge rate, and time in charge depleting and charge sustaining mode. The cumulative charge throughput is defined as -

$$
A h_{t}=\int_{0}^{t}|I(\tau)| d \tau
$$

where $I(t)$ is instantaneous current through the cell, and $A h_{t}$ is the accumulated ampere hour at time $t$. The capacity loss $\left(x_{c}\right)$ or the State Of Health (SOH) of a battery cell as a function of cumulative charge throughput is defined as -

$$
x_{c}(t)=100 \% \times \frac{S(0)-S\left(A h_{t}\right)}{S(0)}
$$

where, $S$ is the capacity of the battery cell (typically measured in Coulomb), which is a function of cumulative charge throughput $A h_{t}$. Other than the accumulated throughput, the time dependency of the capacity fade is also encoded in Ratio, which is defined as follows [17].

$$
\text { Ratio }=\frac{t_{c d}}{t_{c d}+t_{c s}}
$$


Table 1: Summary of aging experiments [17]

\begin{tabular}{lllllll}
\hline$\#$ & $\begin{array}{l}\text { Operating } \\
\text { Mode }\end{array}$ & Ratio & $\begin{array}{c}\text { SOC } C_{\text {min }} \text { Charging } \\
\text { rate }\end{array}$ & $\begin{array}{l}\text { Tempera- } \\
\text { ture }\left[{ }^{0} \mathrm{C}\right]\end{array}$ & $\begin{array}{l}\text { Charge } \\
\text { throughput } \\
{[A h]}\end{array}$ \\
\hline 1 & & & & & \\
2 & & 1 & 45 & $\mathrm{C} / 3$ & 30 & 12364 \\
3 & & 1 & 35 & $\mathrm{C} / 3$ & 30 & 13958 \\
4 & & 1 & 25 & $\mathrm{C} / 3$ & 30 & 12713 \\
5 & Charge & 1 & 45 & $3 \mathrm{C} / 2$ & 30 & 24361 \\
6 & depleting & 1 & 35 & $3 \mathrm{C} / 2$ & 30 & 25353 \\
7 & & 1 & 25 & $3 \mathrm{C} / 2$ & 30 & 27597 \\
8 & & 1 & 45 & $5 \mathrm{C}$ & 30 & 24292 \\
9 & & 1 & 35 & $5 \mathrm{C}$ & 30 & 28141 \\
\hline 10 & \multirow{2}{*}{ Charge } & 0 & 45 & - & 30 & 30704 \\
11 & sustaining & 0 & 35 & - & 30 & 17477 \\
12 & & 0 & 25 & - & 30 & 20080 \\
\hline 13 & & $1 / 2$ & 35 & $3 \mathrm{C} / 2$ & 10 & 19385 \\
14 & & $1 / 2$ & 35 & $3 \mathrm{C} / 2$ & 30 & 20719 \\
15 & Mixed & $1 / 4$ & 35 & $3 \mathrm{C} / 2$ & 30 & 23087 \\
16 & & $1 / 2$ & 35 & $3 \mathrm{C} / 2$ & 45 & 21316 \\
\hline
\end{tabular}

where, $t_{c d}$ is the time in charge depleting mode and $t_{c s}$ is the time in charge sustaining mode. During the experimental campaign it was found that at low charging rate, the capacity fade is not sensitive to the charging rate, so it is not explicitly modeled here.

Now, the semi-empirical capacity fade model can be written as [17] -

$$
x_{c}\left(A h_{t}\right)=a_{c}\left(S O C_{m i n}, \text { Ratio }\right) e^{-E_{a c} / R_{g} T}\left(A h_{t}\right)^{y}
$$

where, $T$ is cell skin temperature, $S O C_{\min }$ is the minimum SOC, $E_{a c}$ is the activation energy, $R_{g}$ is the ideal gas constant, and $y \in(0,1]$ is a calibration 
variable. The function $a_{c}\left(S O C_{\min }\right.$, Ratio $)$ has the form -

$$
a_{c}\left(S O C_{\text {min }}, \text { Ratio }\right)=a_{1}+a_{2}\left(\text { Ratio }^{a_{3}}+a_{4}\left(S O C_{\text {min }}-S O C_{0}\right)^{c}\right.
$$
calibration variables at known $S O C_{0}=0.25$ and $c=3$.

In order to calibrate the capacity fade model dependence on the stress factors, the experimental data obtained during an aging campaign conducted on pouch cells with composite NMC-LMO positive electrode and carbon graphite 175 negative electrode is used. The aging campaign is summarized in table 1. Experiment 15 from table 1 is used for validation of the model and predictive capabilities of the model, hence, it is not included in the calibration process. All the other experiments are used for calibrating the parameters. The parameter values obtained after calibration process are given in table 2 .

Table 2: Calibrated model parameter values.

\begin{tabular}{ccc}
\hline Parameter & Value & Unit \\
\hline$y$ & 0.49 & \\
$a_{1}$ & 280.734 & \\
$a_{2}$ & 454.563 & \\
$a_{3}$ & 0.492 & \\
$a_{4}$ & 10297.97 & \\
$E_{a c}$ & 22406 & $\mathrm{Jmol}^{-1}$ \\
$S O C_{0}$ & 0.25 & \\
$c$ & 3 & \\
$R_{g}$ & 8.314 & $\mathrm{JK}^{-1} \mathrm{~mol}^{-1}$ \\
\hline
\end{tabular}

180

\subsubsection{Uncertainty analysis}

A uncertainty analysis is done using Monte Carlo (MC) analysis to determine the accuracy of the aging model for prognosis. 100 nodes are randomly generated 
in the parameter space using normal distribution with mean equal to nominal parameter values and standard deviation of $5 \%$ of the nominal value. The 100 models thus generated are simulated and the results are compared with the experimental data obtained from experiment 15. The standard deviation and mean are shown in Fig. 2b. It can be seen that the uncertainty in the prediction is not acceptable for long term prognosis.

A sensitivity analysis is performed on the aging model to identify the parameters that affect prognosis. The sensitivity analysis is done using the numerical perturbation method. The normalized sensitivity is defined as -

$$
\operatorname{Sen}(\alpha)=\frac{\left.A h_{\alpha+\delta \alpha}\right|_{\left[x_{c}=0.2\right]}-\left.A h_{\alpha-\delta \alpha}\right|_{\left[x_{c}=0.2\right]}}{2 \delta \alpha}
$$

where $\alpha$ without a subscript is one of the aging model parameters -

$$
\left[\begin{array}{llllll}
y & a_{1} & a_{2} & a_{3} & a_{4} & E_{a c}
\end{array}\right]
$$

$\delta \alpha$ is a perturbation in the parameter, and $S e n$ is the sensitivity metric. The sensitivity metric provides the change in the cumulative charge throughput required to reach $80 \%$ of the $\mathrm{SOH}$, for a small change in the aging model parameter.

Fig. 2c shows the comparison between absolute sensitivities with respect to all the parameters. It can be seen that only parameters $y$ and $a_{3}$ have significant effect on the $\mathrm{SOH}$ prediction.

\subsubsection{Parameter estimation}

In order to demonstrate that the online parameter estimation improves predictive capabilities of the model, we used four different online parameter estimation algorithms namely - 1) Extended Kalman Filter (EKF), 2) Unscented Kalman Filter (UKF), 3) Particle Filter (PF) and 4) Extended Kalman Particle Filter (EKPF) [23]. For parameter estimation, the capacity measurements available from experiment 15 are used as measured capacity. In order to improve the estimation, we resampled the data at smaller sampling period of 0.8 [kAh]. Fig. 3 and table 3 show that the models obtained after parameter estimation have better prediction Root Mean Square Error(RMSE). For estimation and 


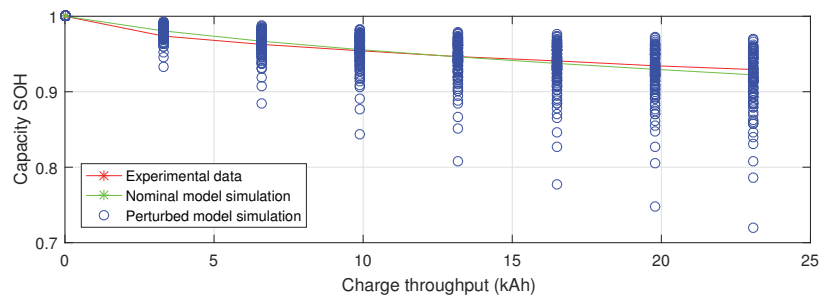

(a) Monte-Carlo analysis

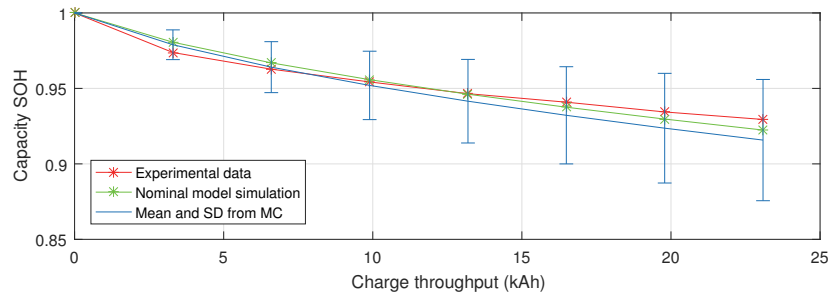

(b) Mean and standard deviation from MC analysis

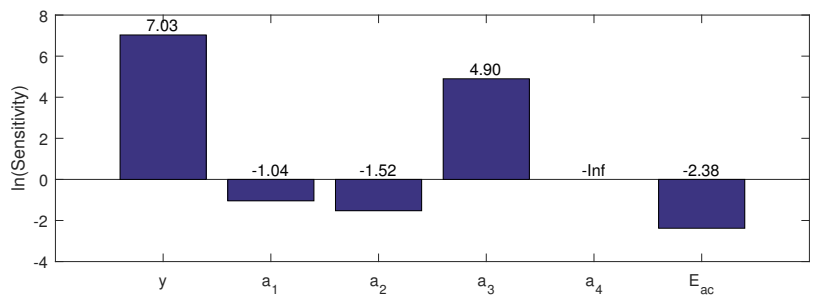

(c) Sensitivity of the model with respect to each parameter variation separately

Figure 2: Uncertainty analysis of the aging model

prediction, the re-sampled experimental data is divided into two zones, estimation period and prediction validation period. First 23 data points, i.e. 0 to 17.6 [kAh] charge throughput, are used for estimation and last 6 data points 17.6 to $22.4[\mathrm{kAh}]$ of charge throughput are used to validate the predictions made by the original model and the estimated model. Only parameters $a_{1}, a_{2}, a_{3}$ and $a_{4}$ are used for the estimation. Although parameter $y$ has the maximum effect on the accuracy of the model, the value of $y$ is determined from the electrochemical model. The value of $y$ should be close to 0.5 and hence it is not really a calibration parameter. Similar argument is true for $E_{a c}$. 


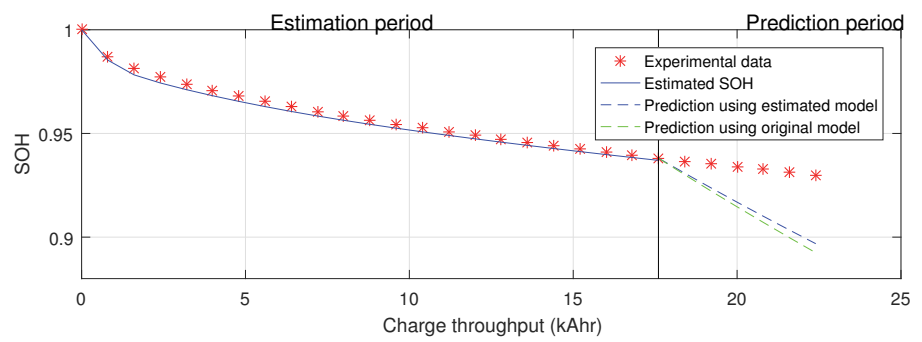

(a) EKF

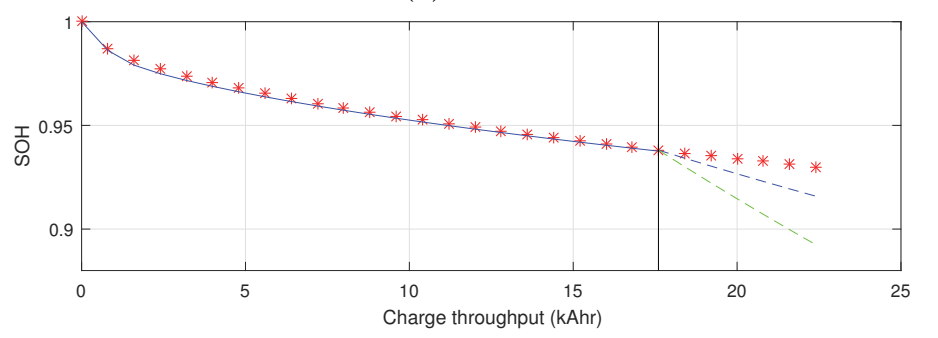

(b) UKF

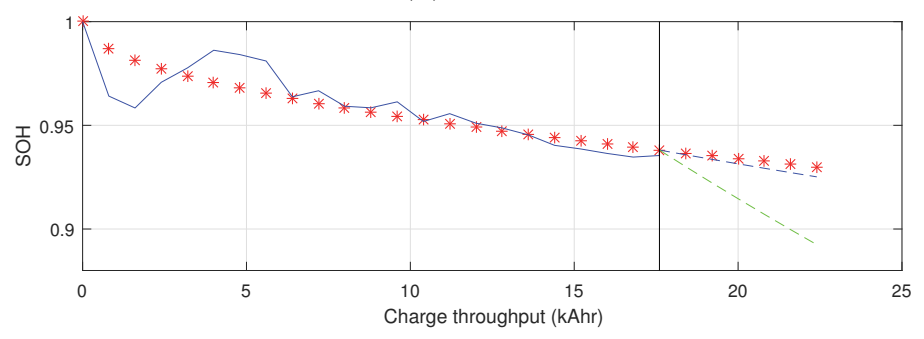

(c) $\mathrm{PF}$

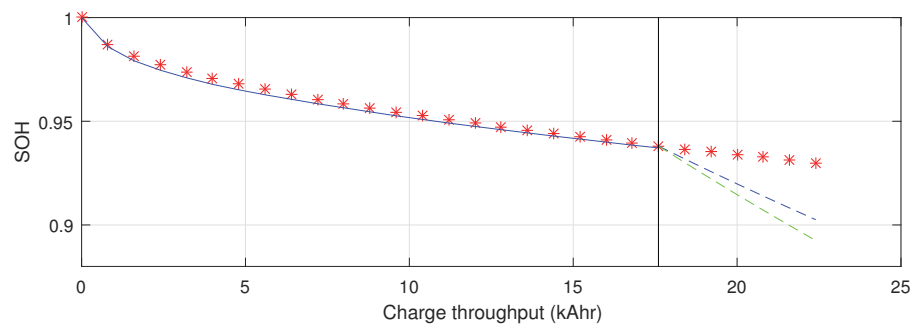

(d) EKPF

Figure 3: Comparison between $\mathrm{SOH}$ predictions made by the original model, estimated model and the experimental data. 
Table 3: Estimation and prognosis results for all four estimation algorithms.

\begin{tabular}{lrrrr}
\hline & EKF & UKF & PF & EKPF \\
\hline Estimation RMSE (10-3) & 9.67 & 6.22 & 46.3 & 2.81 \\
Prediction RMSE (10-2) & 5.33 & 2.28 & 0.784 & 3.34 \\
Improvement in prediction [\%] * & 11.7 & 62.2 & 87 & 44.7 \\
$a_{1}$ (final) & 170.3 & 169.6 & 68.5 & 166.2 \\
$a_{2}$ (final) & 295.6 & 402.9 & 183.6 & 250.7 \\
$a_{3}$ (final) & 0.13 & 1.2 & 0.7 & 0.64 \\
$a_{4}$ (final) & 10293 & 10292 & 9400 & 10304 \\
\hline
\end{tabular}

${ }^{1}$ Improvement as compared with the original model without any estimation.

The prediction RMSE without any parameter estimation is $6.04 \times 10^{-2}$.

During the prediction period, the RMSE between the capacity measurements (experimental data) and $\mathrm{SOH}$ predictions made by the original model is $6.04 \times$ $10^{-2}$. It can be observed from table 3 that the model parameters obtained at the end of the estimation period provide a model with better prognosis capabilities. It can be noticed from the table 3 that the estimation RMSE is more than the prediction RMSE. This is due to the fact that the estimation takes time to converge to the true values of parameters, whereas the model parameters for prediction period are already estimated.

In conclusion, the model calibrated using laboratory experiments can be significantly improved by performing online parameter estimations. This result for single cell motivated us to leverage the online parameter estimation method to apply for battery packs.

\section{Aging Model of a Battery Pack}

In this section, a framework is developed for pack level prognosis using the cell level aging model. A probabilistic framework is proposed to derive the aging model for battery packs based on the typically available measurements or estimates in a PHEV. 
For a common battery management system such as the one shown in [24], cell (or module) level voltage and temperature is measured for battery management. Integrated pack current is also available. Since we consider string cells interconnection as shown in Fig. 4, the current of every module in series is simply equal to the current of the pack. The physical variables of the cells $z_{i}, i \in[N]$, can all be measured and the SOC of all the cells can be estimated. The estimation on $\mathrm{SOC}_{i}, i \in[N]$ is required for cell balancing control, and for prognosis. However, cell level SOC is not required for prognosis of the pack, but only pack level SOC estimation is sufficient.

Among many works dealing with the SOC estimation [25, 26, 27, 28, 29], several of them further use the estimated SOC to compute the capacity instead of the aging model in Eq. (1) [28]. However, all those estimations on the capacity only regard the capacity as a constant variable. The assumption is valid for many applications due to the slow degradation of the capacity. Since our objective is updating the model that captures the slow aging of the capacity, a measurement or estimation on the slow varying capacity is needed. Hence, we will extend the existing SOC estimation framework and incorporate the model based estimation on the time varying capacity. A battery pack SOC estimation algorithm using lumped parameter model of the pack and dual filters was proposed by Zhang et al. [30] and a machine learning based method was proposed in [31]. The proposed dual SOC and capacity estimation method can be considered as a direct extension of [29]. In [29], an analytical method is proposed to estimate SOC. The open circuit voltage (OCV), $V_{o c}$ is first analytically solved using the sequence of measurements of terminal voltage and current. The SOC is then analytically derived by $V_{o c}$ and the OCV-SOC curve. With the analytical solution of $V_{o c}$, we modify the SOC estimation method in [29] into dual SOC 
and time-varying capacity estimation in the following

$$
\left\{\begin{array}{l}
\mathrm{SOC}^{k+1}=\mathrm{SOC}^{k}+\frac{\Delta I^{k}}{X_{c}^{k}}+w_{x}^{k}, \\
V_{o c}^{k}=\eta^{k} \mathrm{SOC}^{k}+w_{y}^{k}
\end{array}\right.
$$
one is unknown. The aging model is updated whenever the capacity estimation from the measurement is made. As illustrated in Fig. 4, all SÔC, $\hat{X}_{c}^{k}$, and 


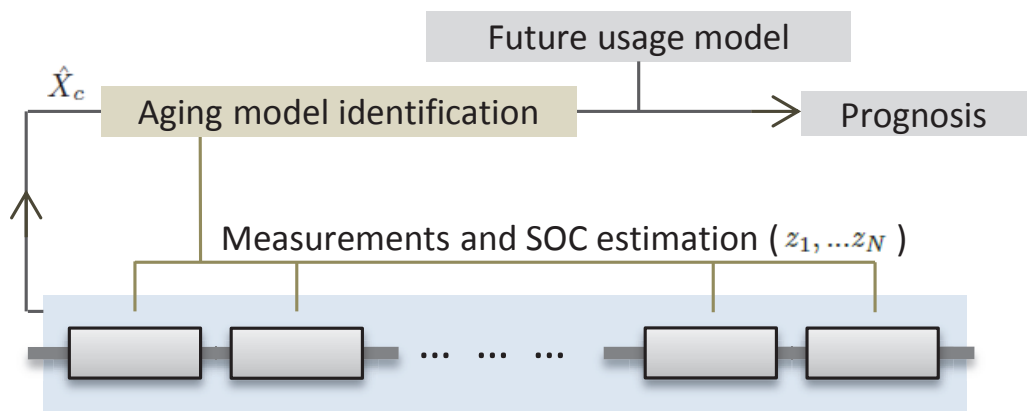

Figure 4: The framework for prognosis. First the online measurements or estimation are made on some physical variables. The measurements are then used to identify the aging model for prognosis purpose.

$z_{i}$ (excluding $\mathrm{SOC}_{i}$ ) served as the reference for the identification of the aging model. For convenience, we will define $z_{i}=\left[T_{i}, V_{i}, I_{i}\right]$ as the physical variables to distinguish the directly measured variables from the estimated ones.

\subsection{Probabilistic Method}

Given the aging model of cells in Eq. (1) and cell-pack relation of the percentage capacity loss, the aging model of the pack is developed. Due to the manufacturing difference, every cell in the pack may have different aging properties. The heterogeneous aging among cells is characterized by $\alpha$. It is assumed that the statistical data reflecting the manufacturing differences is available so that the distribution function of $\alpha$ is known as a priori. Every cell $j$ has $\alpha_{j}$ initialized to follow the distribution with density function $D^{0}$. The probability distribution of every cell $D_{j}$ can be updated as the physical variables SÔC, $\hat{X}_{c}^{k}$, and $z_{i}$ are measured or estimated. Given SÔC, $\hat{X}_{c}^{k}$, and $z_{i}$, the probability for cell $i$ being the most aged at time $k, P\left(X_{c}^{k}=f_{c}\left(\left[\mathrm{SO}^{k}, z_{i}^{k}\right], \alpha_{i}^{k}, k\right)\right)$, can be calculated. By viewing the capacity of cell $i, x_{c, i}^{k}=f_{c}\left(\left[\mathrm{SOCC}^{k}, z_{i}^{k}\right], \alpha_{i}^{k}, k\right)$ as a new random variable originated from random parameters $\alpha_{i}^{k}$, we have 


$$
\begin{aligned}
p_{i}^{k} & \triangleq P\left(X_{c}^{k}=f_{c}\left(\left[\mathrm{SOC}^{k}, z_{i}^{k}\right], \alpha_{i}^{k}, k\right)\right) \\
& =\frac{1}{Z(k)} \int d \alpha d w\left(\mathbb{1}_{\mathcal{A}}(\alpha, \omega) \Omega(w) D_{i}^{k-1}\right),
\end{aligned}
$$

285

where $\Omega$ as the probability density function of $w$,

$$
\mathcal{A}=\left\{(\alpha, \omega) \mid \hat{X}_{c}^{k}=f_{c}\left(\left[\operatorname{SÔC}^{k}, z_{i}^{k}\right], \alpha, k\right)+w\right\}
$$

and $\mathbb{1}$ is an indicator function defined as -

$$
\mathbb{1}_{\mathcal{A}}(\alpha, \omega)= \begin{cases}1 & (\alpha, \omega) \in \mathcal{A} \\ 0 & (\alpha, \omega) \notin \mathcal{A}\end{cases}
$$

The normalization factor $Z(k)$ is defined such that

$$
\sum_{i \in[N]} p_{i}^{k}=1
$$

The precise calculation of $p_{i}^{k}$ also needs to consider $w_{s}$. We omit the details for simplicity. Notice that instead of cell level $\mathrm{SOC}_{i}$, only the pack SOC is sufficient in calculating $p_{i}^{k}$ because $\mathrm{SOC}=\mathrm{SOC}_{i}$ if $X_{c}=x_{c, i}$ and the battery pack has passive balance control. With the probability $p_{i}^{k}, X_{c}^{k}=x_{c, i}^{k}$ and the density function of cell is updated by $\hat{X}_{c}^{k}$, which leads to the following rule for the update of $D_{i}^{k}$

$$
D_{i}^{k}=\left(1-p_{i}^{k}\right) D_{i}^{k-1}+p_{i}^{k} \Omega_{\alpha_{i}}^{k} .
$$

where $\Omega_{\alpha_{i}}^{k}$ is the probability density function of $\alpha_{i}$ such that $f_{c}\left(\left[\mathrm{SOCC}^{k}, z_{i}^{k}\right], \alpha_{i}^{k}, k\right)+$ $w=\hat{X}_{c}^{k}$. The Eq. (14) can be explained as follows - the probability of not updating the probability density function of $\alpha_{i}$ is the probability that cell $i$ is not the most aged cell. The probability that an update in the probability distribution is applied is the same as the probability that cell $i$ is the most aged. The updated probability density function is given by $\Omega_{\alpha_{i}}^{k}$. The characterization of $\Omega_{\alpha_{i}}^{k}$ will be discussed later. 
With sufficient iterations, the battery pack aging is well defined by $D_{i}^{k}$ and Eq. (1-2) for the prognosis purpose. Let $z_{i 0}$ be the prediction on future input and $\bar{X}_{c}$ be the threshold capacity such that if $X_{c} \geq \bar{X}_{c}$, the battery pack is considered reaching EOL. The EOL of the pack at time $l>k$, i.e., $X_{c}^{l} \geq \bar{X}_{c}$, is given by

$$
P\left(X_{c}^{l} \geq \bar{X}_{c}\right)=\sum_{i \in[N]} p_{i}^{k} P\left(f_{c}\left(z_{i 0}^{l}, \alpha_{i}^{k}, l\right) \geq \bar{X}_{c}\right)
$$

295 updates of the distribution of $\alpha_{i}^{k}$ by $\Omega_{\alpha_{i}}^{k}$ can then be interpreted as a process of finding the true $\alpha_{i}$ from the measurement update. The idea is that, instead of finding the nontrivial distribution function $\Omega_{\alpha_{i}}^{k}$, we find an optimal $\alpha_{i}^{*}$ that minimizes the difference between $\hat{X}_{c}$ and $f_{c}\left(z_{i}, \alpha_{i}^{*}, k\right)$. To further illustrate the 315

where $\alpha_{i}^{k}$ follows the distribution $D_{i}^{k}$, which is written as $\alpha_{i}^{k} \sim D_{i}^{k}$. We will show that Eq. (15) provides a valueable information for prognosis with only few iteration updates on $D_{i}^{k}$ and $p_{i}^{k}$.

Although the probabilistic method has several advantages and characterizes battery pack aging, the update of $D_{i}^{k}$ in Eq. (14) involves non-trivial computation in finding $\Omega_{\alpha_{i}}^{k}$. Especially when the number of aging model parameters is large, it is impossible to analytically characterize $\Omega_{\alpha_{i}}^{k}$ for a general $f_{c}(\cdot)$. An approximation on $\Omega_{\alpha_{i}}^{k}$ is needed for the real implementation of the proposed probabilistic framework.

\subsection{Approximation of $\Omega_{\alpha_{i}}^{k}$}

The probabilistic framework for prognosis proposed in the last section is composed of three parts, including updates on $p_{i}^{k}, D_{i}^{k}$ and computes $P\left(X_{c}^{l} \geq\right.$ $\left.\bar{X}_{c}\right)$. The complexity issue lies in the second step where $\Omega_{\alpha_{i}}^{k}$ may not be possibly derived. An alternative method to approximate $\Omega_{\alpha_{i}}^{k}$ is therefore proposed in this subsection.

As stated earlier, we assume that the aging model is time invariant. The how $D_{i}^{k}$ depends on $\Omega_{\alpha_{i}}^{j}$ for $j=1, \cdots, k$, we write Eq. (14) equivalently to the following equation - 


$$
D_{i}^{k}=\prod_{j=1}^{k}\left(1-p_{i}^{j}\right) D^{0}+\sum_{j \in[k-1]}\left(\prod_{l=1}^{k-j}\left(1-p_{i}^{k-l}\right)\right) p_{i}^{j} \Omega_{\alpha_{i}}^{j}+p_{i}^{k} \Omega_{\alpha_{i}}^{k} .
$$

Eq. (16) is derived by substituting $D_{i}^{j}$ recursively by Eq. (14) for all $j<k$. The first part of Eq. (16) represents the initial distribution that is not subject to updates. We propose to replace the remaining part by

$$
\left(1-\prod_{j \in[k]}\left(1-p_{i}^{j}\right)\right) \mathbb{1}_{\left\{\alpha_{i}=\alpha_{i}^{*}\right\}}
$$

where

$$
\alpha_{i}^{*}=\operatorname{argmin}_{\alpha} \sum_{j \in[k]} p_{i}^{j}\left|\hat{X}_{c}^{j}-f_{c}\left(z_{i}^{j}, \alpha, j\right)\right| .
$$

In the optimization problem shown in Eq. (18), $\alpha_{i}^{*}$ minimizes the difference between the estimated cell capacity and the measured pack capacity weighted by the probability of being the most aged cell $i$. Since we assume that the real aging model parameters are not subject to change over time, calculation of single value of $\alpha_{i}^{*}$ instead of its distribution (replacing Eq. (16) by Eq. (17)) is considered as a viable approximation. Note that in Eq. (18), we ignore the unbiased Gaussian measurement noise for simplicity in solving the optimization problem. Combining Eq. (16-18), the new update law on the distribution of $\alpha_{i}$ is given as follows

$$
D_{i}^{k}=\prod_{j=1}^{k}\left(1-p_{i}^{j}\right) D^{0}+\left(1-\prod_{j=1}^{k}\left(1-p_{i}^{j}\right)\right) \mathbb{1}_{\left\{\alpha_{i}=\alpha_{i}^{*}\right\}} .
$$

Despite the proposed approximation method manages to update the distribution that is originally computationally intractable, the optimization in Eq. (18) may remain computationally heavy, especially when $N$ and $n$ are large. However, the computation is not necessary to be completed immediately after the 
measurements because the capacity measurements are available only at slow sampling rates. It takes only several minutes to finish all the computations for a battery pack example with 100 cells, which is completely acceptable for prognosis purpose.

The overall aging model identification framework for the battery pack is summarized as follows

1. Initialization: Set $\alpha_{i} \sim D^{0}, \forall i \in[N], k=0$

2. Measurement Updates: Given the measurement $z_{i}$

(a) Update $S \hat{O} C^{k}$ : Using Kalman filter on Eq. (11).

(b) Update $p_{i}^{k}$ by Eq. (12).

(c) Compute $\alpha_{i}^{*}$ by Eq. (18).

(d) Approximate $D_{i}^{k}$ by Eq. (19).

(e) Set $k=k+1$. Repeat the measurement updates.

3. Prognosis: Given $l>k, p_{i}^{k}, D_{i}^{k}$,

(a) Predict $z_{i}^{m}$ for $m$ such that $k<m \leq l$ using historic data or other on-board driving pattern recognition methods

(b) Compute the probability of reaching EOL by Eq. (15).

\section{Simulation Studies}

A 100 cell battery pack model is used to demonstrate the prognosis algorithm presented in the previous section. The aging of the cells follow the model in [17]. The simulation results indicate that the proposed method effectively estimates the aging model parameters, which leads to an accurate prediction of the capacity fade for prognosis.

The aging model used is taken from [17] as explained in section 2 .

$$
x_{c, i}^{k}=\left(a_{1 i}+a_{2 i}\left(\text { Ratio }^{k}\right)^{a_{3 i}}+a_{4 i}\left(S O C_{m i n}-S O C_{0}\right)^{c}\right) \cdot \exp \left(\frac{-E_{a c}}{R_{g} T^{k}}\right) A h_{k}^{y_{i}},
$$

where $\alpha_{i}=\left[a_{1 i}, a_{2 i}, a_{3 i}, a_{4 i}\right]$ are aging model parameters to be estimated. $i$ is the cell index and $k$ is the time step index. The values of the aging model 
parameters are listed in Table 4 and $S O C_{\min }=0.25$ and $S O C_{0}=0.27$ are chosen.

Table 4: Selected Variables for the simulation

\begin{tabular}{lccccccc}
\hline & $a_{1}$ & $a_{2}$ & $a_{3}$ & $a_{4}$ & $y$ & $E_{a c}$ & Ratio \\
\hline Unit & $(-)$ & $(-)$ & $(-)$ & $(-)$ & $(-)$ & $(\mathrm{J} / \mathrm{mol})$ & $(-)$ \\
Mean & 137 & 420 & 0.34 & 9610 & 0.48 & 22406 & 0.84 \\
Variance & 24.66 & 75.6 & 0.16 & 1300 & $(-)$ & $(-)$ & 0.02 \\
\hline
\end{tabular}

360

Since the proposed aging model parameter estimation framework estimates $\alpha_{i}, i \in[N]$, the computational complexity can be reduced a lot when the dimension of $\alpha_{i}$ gets smaller. In this simulation study we estimate parameters $a_{i}$ $\forall i=[1,2,3,4]$ using the proposed approach. As mentioned in section 2, the value of the parameter $y$ is derived from the electro-chemical aging model of the battery, and hence it is not used for estimation. The simulation is divided into two parts. First, we simulate the battery pack aging by assigning $\alpha_{i}$ to every cell in the pack based on the distribution listed in Table 4 . The current is obtained from an artificially generated realistic driving cycle data based on [33]. The temperature is generated randomly, which follows the normal distribution with mean $25\left({ }^{\circ} \mathrm{C}\right)$ and variance $3\left({ }^{\circ} \mathrm{C}\right)$. Some cells have slightly higher average temperature than the others to account the temperature heterogeneity in the pack. The time horizon is 60 days. The capacity data generated from this simulation is considered as reference case. In the second part, the algorithm presented in previous section is implemented for parameter estimation and prognosis. We now use the capacity and SOC are available from the reference case at a time interval of every two days. An error less than $2 \%$ is introduced in the measurements. The average of $z_{i}$ is also available at higher frequency, so the updated capacity prediction from Eq. (12), (18), and (19) can be made in every 380 two days. Consider at the $24^{t h}$ day, a prediction of the pack capacity at day 60 is made.

As illustrated in Fig. 5a, the proposed framework shows that with high 


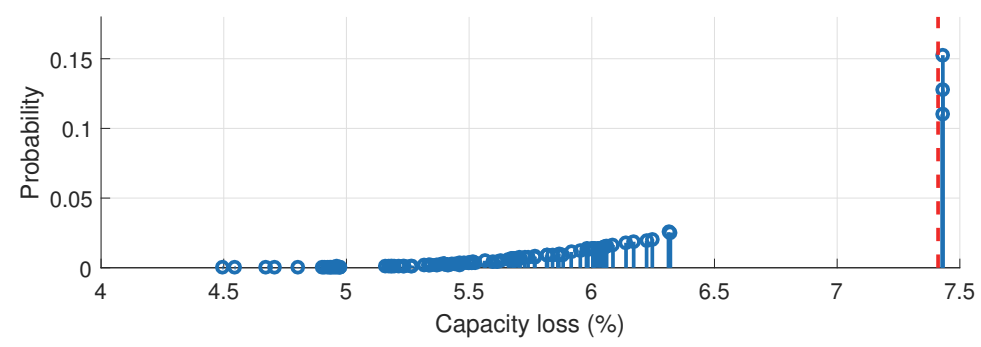

(a) The predicted capacity loss at day 60. The red star is the real capacity loss and each blue circle represents the estimated capacity loss of one cell if the cell is the weakest one.

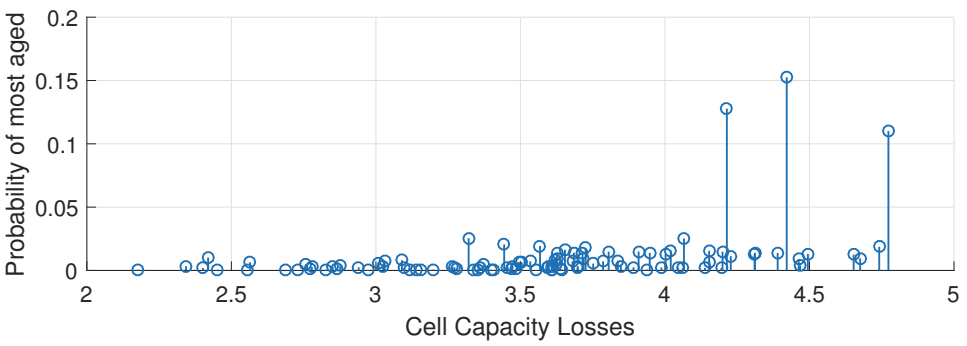

(b) Capacity loss of the cells verses its probability of being most aged at day 24.

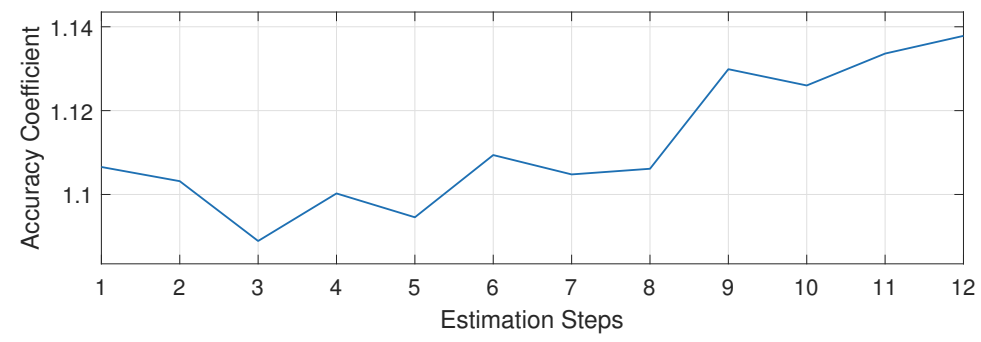

(c) The evolution of accuracy coefficient $\eta_{c}$ with the iterations.

Figure 5: Results of the simulation study: comparison between estimated capacity and real capacity, and probability of cells being the most aged cell.

probability, the capacity loss is $7.5 \%$, which is close to the capacity loss obtained from the reference case. Note that in Eq. (12), a high dimensional integral is needed to update $p_{i}^{k}$ for all $i$. We approximate the integral by Monte Carlo (MC) method with 7000 randomly generated nodes. The set for the indicator function 
in Eq. (12), $\mathcal{A}=\left\{\hat{X}_{c}^{k} \mid \hat{X}_{c}^{k}=f_{c}\left(\left[\mathrm{SOCC}^{k}, z_{i}^{k}\right], \alpha_{i}, k\right)+w\right\}$, is also approximated by replacing the strict equality with an inequality with upper and lower bounds defined by an interval- $\left[\hat{X}_{c}^{k}-\epsilon, \hat{X}_{c}^{k}+\epsilon\right]$ for some $0<\epsilon<\hat{X}_{c}^{k}$. Now, the approximation of the set for the indicator function is given by -

$$
\begin{array}{r}
\mathcal{A} \approx\left\{\hat{X}_{c}^{k} \mid \hat{X}_{c}^{k} \in\left[f_{c}\left(\left[\mathrm{SOCC}^{k}, z_{i}^{k}\right], \alpha_{i}, k\right)-\epsilon+w\right.\right. \\
\left.\left., f_{c}\left(\left[\mathrm{SOCC}^{k}, z_{i}^{k}\right], \alpha_{i}, k\right)+\epsilon+w\right]\right\}
\end{array}
$$

The approximation of $p_{i}^{k}$ can be further improved by increasing the number of nodes in the MC integration and by choosing a smaller $\epsilon$ in Eq. (21).

In the proposed method, the probability of one cell to be weakest is calculated. In this simulation study it is demonstrated that the cells with higher capacity losses have relatively large $p_{i}^{k}$. As shown in Fig. 5b, three cells with relatively large capacity losses share around $40 \%$ of probability of being the weakest cell. With small number of updates (12 in the case), the proposed framework successfully identifies the weakest cells. The prognosis will be improved as more measurements become available, hence the EOL prediction will keep on improving as the pack ages further. In order to quantify how the aging model estimation accuracy improves as more updates become available, we define accuracy coefficient as $\eta_{c}=\frac{\sum_{i \in[N]} p_{i}^{k} x_{c, i}^{k}}{\sum_{i \in[N]} x_{c, i}^{k}}$. A larger $\eta_{c}$ implies more accurate estimation on $p_{i}^{k}$. As expected, $\eta_{c}$ grows with the number of iterations as shown in Fig. 5c.

Fig. 6 a shows the evolution of parameters $a_{1}$ and $a_{2}$ as an example though the estimation process and Fig. $6 \mathrm{~b}$ shows evolution of probability of being most aged cell of first three most aged cells.

The accuracy of the aging model depends on the variance of $\alpha_{i}$ and the temperature. When the variance of the temperature is larger than the variance of $\alpha_{i}$, the estimation of $p_{i}$ is more accurate. On the other hand, the uncertainty grows as the variance of the non-measureable $\alpha_{i}$ get larger. Consider the case when the variance of the temperature reduces to $2\left({ }^{\circ} \mathrm{C}\right)$ with the variance of $\alpha_{i}$ being the same. As shown in Fig. 7c, it can be observed that the estimated 


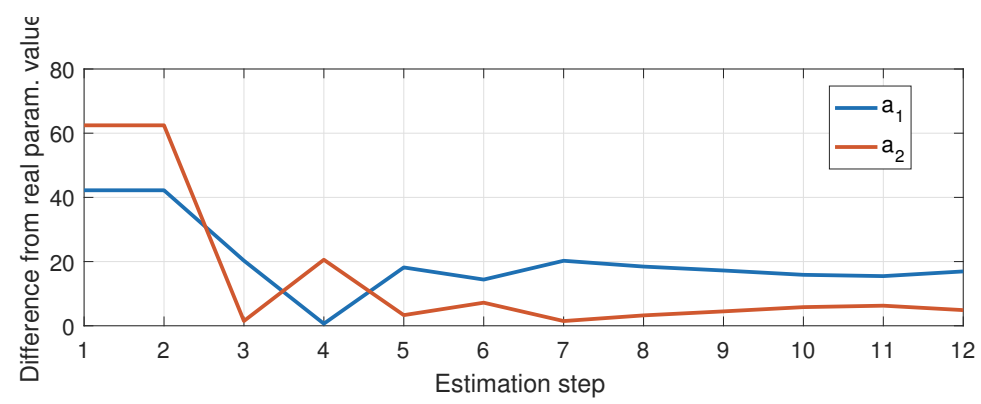

(a) Evolution of the estimation as battery ages

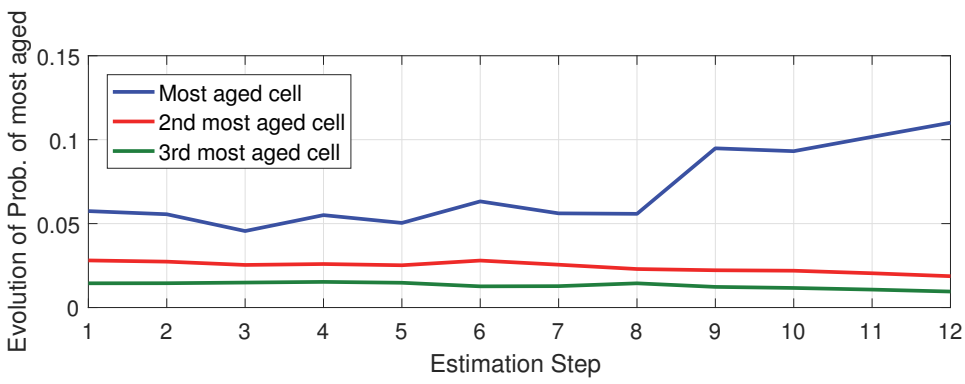

(b) The evolution of accuracy coefficient $\eta_{c}$ with the iterations.

Figure 6: Evolution of aging model parameters and the probability of being most aged cell.

capacity loss has the error less than $2 \%$. However, as shown in Fig. $7 \mathrm{~b}$, there 415 is one relatively not aged cell having a high probability $(\approx 9.5 \%)$ of being the weakest, which indicates the less accuracy compared to the case with the variance of temperature being $3 \%$. In such case, it requires more iterations and updates to identify the weakest cells.

\section{Conclusion}

It is demonstrated using single cell aging data that an aging model alone is not sufficient for prognosis in practice, and that on board aging model parameter estimation is necessary. Hence, a modeling framework is proposed that leverages the aging model of single cell to the prognosis of a battery pack in a probabilistic sense. The proposed framework captures heterogeneity in the cell aging dynamics and shows satisfactory accuracy for prognosis purposes. An 


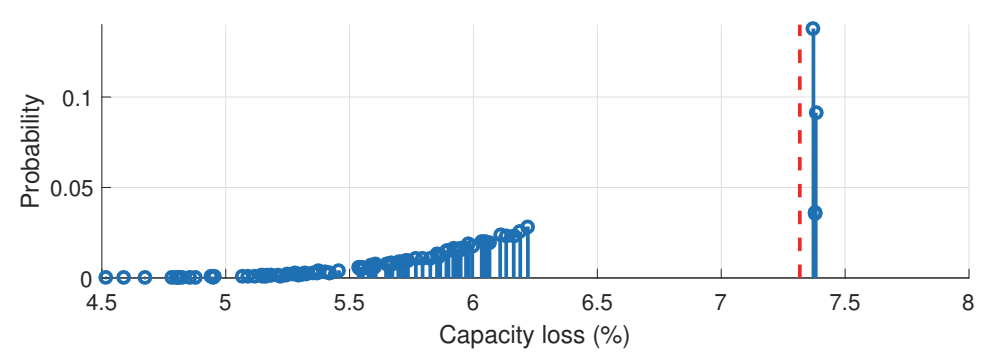

(a) Capacity loss (\%). The variance of temperature: $2\left({ }^{\circ} \mathrm{C}\right)$.

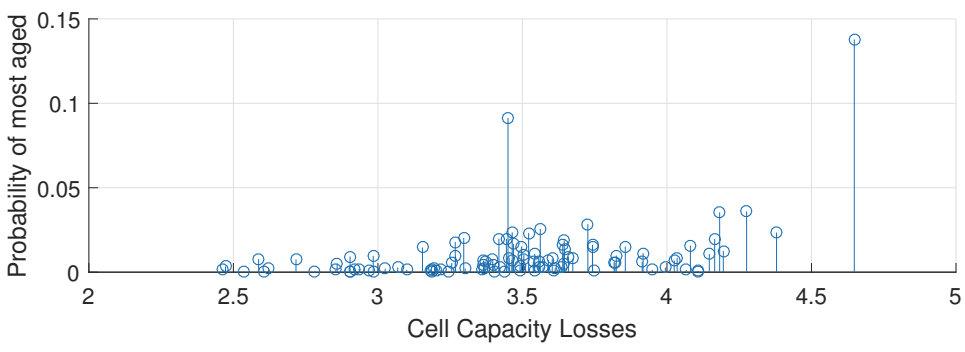

(b) Capacity loss of the cells verses its probability of being most aged at day 24.

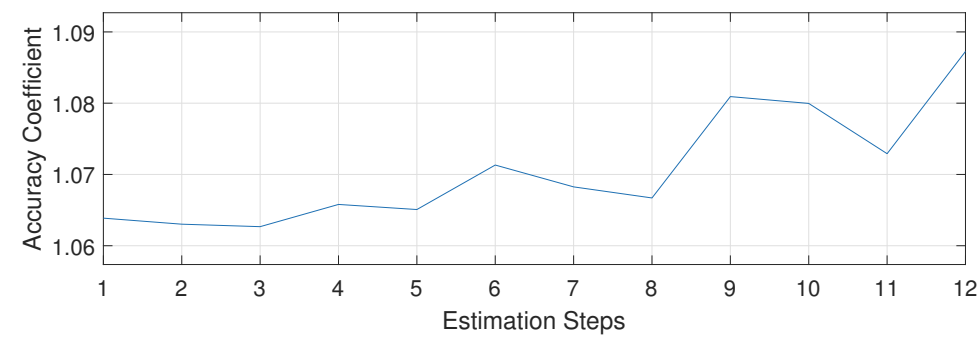

(c) The evolution of accuracy coefficient $\eta_{c}$ with the iterations. The variance of temperature: $2\left({ }^{\circ} \mathrm{C}\right)$.

Figure 7: Results of the simulation study: comparison between estimated capacity and real capacity, and probability of cells being the most aged cell. The variance of temperature is $2\left({ }^{\circ} \mathrm{C}\right)$.

added benefit of the proposed framework is that it, does not put any restriction on the battery chemistry or what type of cell-level aging model is adopted. In addition, the proposed framework provides an indication of which cells (or modules) may be most aged and supervises replacement of those cells. This 
430

ormation can also be vital in rebuilding the pack or servicing. The proposed method is demonstrated to be effective in a simulation study. Experimental validation on the proposed aging model is considered as an important future work.

\section{Acknowledgement} dation under Grant No.1301238

\section{Bibliogrphy}

[1] M. Dubarry, N. Vuillaume, B. Y. Liaw, From single cell model to battery pack simulation for li-ion batteries, Journal of Power Sources 186 (2) (2009) $500-507$.

[2] L. Lu, X. Han, J. Li, J. Hua, M. Ouyang, A review on the key issues for lithium-ion battery management in electric vehicles, Journal of power sources 226 (2013) 272-288.

[3] S. B. Peterson, J. Apt, J. Whitacre, Lithium-ion battery cell degradation resulting from realistic vehicle and vehicle-to-grid utilization, Journal of Power Sources 195 (8) (2010) 2385-2392.

[4] A. Barré, B. Deguilhem, S. Grolleau, M. Gérard, F. Suard, D. Riu, A review on lithium-ion battery ageing mechanisms and estimations for automotive applications, Journal of Power Sources 241 (2013) 680-689.

450

[5] M. Ecker, J. B. Gerschler, J. Vogel, S. Käbitz, F. Hust, P. Dechent, D. U. Sauer, Development of a lifetime prediction model for lithium-ion batteries based on extended accelerated aging test data, Journal of Power Sources 215 (2012) 248-257.

[6] J. Vetter, P. Novák, M. Wagner, C. Veit, K.-C. Möller, J. Besenhard, 455 M. Winter, M. Wohlfahrt-Mehrens, C. Vogler, A. Hammouche, Ageing 
mechanisms in lithium-ion batteries, Journal of power sources 147 (1) (2005) 269-281.

[7] A. Fotouhi, D. J. Auger, K. Propp, S. Longo, M. Wild, A review on electric vehicle battery modelling: From lithium-ion toward lithiumsulphur, Renewable and Sustainable Energy Reviews 56 (2016) 1008 - 1021. doi:http://dx.doi.org/10.1016/j.rser.2015.12.009. URL http://www.sciencedirect.com/science/article/pii/ S1364032115013921

[8] B. Y. Liaw, R. G. Jungst, G. Nagasubramanian, H. L. Case, D. H. Doughty, Modeling capacity fade in lithium-ion cells, Journal of Power Sources 140 (1) (2005) 157 - 161. doi:http: //dx.doi.org/10.1016/j.jpowsour.2004.08.017.

URL http://www.sciencedirect.com/science/article/pii/ S0378775304008808

[9] J. Schmalstieg, S. Kbitz, M. Ecker, D. U. Sauer, A holistic aging model for li(nimnco)o2 based 18650 lithium-ion batteries, Journal of Power Sources 257 (2014) 325 - 334 doi:http: //dx.doi.org/10.1016/j.jpowsour.2014.02.012.

URL http://www.sciencedirect.com/science/article/pii/ S0378775314001876

[10] G. K. Prasad, C. D. Rahn, Model based identification of aging parameters in lithium ion batteries, Journal of Power Sources 232 (2013) $79-85$. doi:http://dx.doi.org/10.1016/j.jpowsour. 2013.01.041.

URL http://www.sciencedirect.com/science/article/pii/ S0378775313000700

[11] G. Suri, S. Onori, A control-oriented cycle-life model for hybrid electric vehicle lithium-ion batteries, Energy 96 (2016) 644 - 653 . doi:http://dx.doi.org/10.1016/j.energy.2015.11.075. 
URL

http://www.sciencedirect.com/science/article/pii/

[12] A. Cordoba-Arenas, S. Onori, G. Rizzoni, A control-oriented lithium-ion battery pack model for plug-in hybrid electric vehicle cycle-life studies and system design with consideration of health management, Journal of Power Sources 279 (2015) 791-808.

[13] W. Waag, S. Käbitz, D. U. Sauer, Experimental investigation of the lithium-ion battery impedance characteristic at various conditions and aging states and its influence on the application, Applied Energy 102 (2013) 885-897.

[14] R. L. Hartmann II, An aging model for lithium-ion cells, Ph.D. thesis, The University of Akron (2008).

[15] Y. Wang, D. Yang, X. Zhang, Z. Chen, Probability based remaining capacity estimation using data-driven and neural network model, Journal of Power Sources 315 (2016) 199 - 208. doi:http://dx.doi.org/10.1016/j.jpowsour.2016.03.054. S0378775316302543

[16] J. Wu, C. Zhang, Z. Chen, An online method for lithium-ion battery remaining useful life estimation using importance sampling and neural networks, Applied Energy 173 (2016) 134 - 140. doi:http://dx.doi.org/10.1016/j.apenergy.2016.04.057. URL http://www.sciencedirect.com/science/article/pii/ S0306261916304846

[17] A. Cordoba-Arenas, S. Onori, Y. Guezennec, G. Rizzoni, Capacity and power fade cycle-life model for plug-in hybrid electric vehicle lithium-ion battery cells containing blended spinel and layered-oxide positive electrodes, Journal of Power Sources 278 (2015) 473-483. 
[18] M. Todinov, Necessary and sufficient condition for additivity in the sense of the PalmgrenMiner rule, Computational Materials Science 21 (1) (2001) 101-110. doi:10.1016/S0927-0256(00)00221-4.

[19] Y. Hua, A. Cordoba-Arenas, N. Warner, G. Rizzoni, A multi time-scale state-of-charge and state-of-health estimation framework using nonlinear predictive filter for lithium-ion battery pack with passive balance control, Journal of Power Sources 280 (2015) 293-312.

[20] A. C. Cordoba Arenas, Aging propagation modeling and state-of-health assessment in advanced battery systems, Ph.D. thesis, The Ohio State University (2013).

[21] B. Scrosati, J. Garche, W. Tillmetz, Advances in Battery Technologies for Electric Vehicles, Woodhead Publishing, 2015.

[22] P. Tulpule, C.-Y. Chang, G. Rizzoni, Li-ion cell aging model online parameter estimation for improved prognosis, in: Dynamic systems and control conference (DSCC), ASME, 2016.

[23] D. Simon, Optimal state estimation: Kalman, H infinity, and nonlinear approaches, John Wiley \& Sons, 2006.

[24] T. Instruments, Battery management guide, Texas Instruments.

[25] G. L. Plett, Extended kalman filtering for battery management systems of lipb-based hev battery packs: Part 2. modeling and identification, Journal of power sources 134 (2) (2004) 262-276.

535

[26] G. L. Plett, Extended kalman filtering for battery management systems of lipb-based hev battery packs: Part 3. state and parameter estimation, Journal of power sources 134 (2) (2004) 277-292. 
[27] B. Balasingam, G. Avvari, B. Pattipati, K. Pattipati, Y. Bar-Shalom, A robust approach to battery fuel gauging, part i: Real time model identification, Journal of Power Sources 272 (2014) 1142-1153.

[28] B. Balasingam, G. Avvari, B. Pattipati, K. Pattipati, Y. Bar-Shalom, A robust approach to battery fuel gauging, part ii: Real time capacity estimation, Journal of Power Sources 269 (2014) 949-961.

[29] X. Tang, X. Mao, J. Lin, B. Koch, Li-ion battery parameter estimation for state of charge, in: American Control Conference (ACC), IEEE, 2011, pp. 941-946.

[30] X. Zhang, Y. Wang, D. Yang, Z. Chen, An on-line estimation of battery pack parameters and state-of-charge using dual filters based on pack model, Energy 115, Part 1 (2016) 219 - 229. doi:http://dx.doi.org/10.1016/j.energy.2016.08.109.

URL http://www.sciencedirect.com/science/article/pii/ S0360544216312312

[31] Y. Wang, D. Yang, X. Zhang, Z. Chen, Probability based remaining capacity estimation using data-driven and neural network model, Journal of Power Sources 315 (2016) 199 - 208. doi:http://dx.doi.org/10.1016/j.jpowsour.2016.03.054.

URL http://www.sciencedirect.com/science/article/pii/ S0378775316302543

[32] B. Pattipati, B. Balasingam, G. Avvari, K. Pattipati, Y. Bar-Shalom, Open circuit voltage characterization of lithium-ion batteries, Journal of Power Sources 269 (2014) 317-333.

[33] S. Midlam-Mohler, S. Ewing, V. Marano, Y. Guezennec, G. Rizzoni, Phev fleet data collection and analysis, in: Vehicle Power and Propulsion Conference, 2009. VPPC'09. IEEE, IEEE, 2009, pp. 1205-1210. 